\title{
Correction to: Management of the Patient with Heart Failure and an Implantable Pulmonary Artery Hemodynamic Sensor
}

\author{
Jacob Abraham ${ }^{1} \cdot$ Patrick J. McCann ${ }^{2}$ - Maya E. Guglin ${ }^{3}$ - Arvind Bhimaraj ${ }^{4} \cdot$ Terrie-Ann S. Benjamin $^{5}$. \\ Monique R. Robinson ${ }^{6} \cdot$ Orvar T. Jonsson $^{7}$. Scott C. Feitell ${ }^{8} \cdot$ Kunjan A. Bhatt $^{9} \cdot$ Mosi K. Bennett $^{10}$ - J.T. Heywood ${ }^{11}$. \\ on behalf of Hemodynamic Frontiers in Heart Failure (HF2) Investigators
}

Published online: 18 September 2020

(C) Springer Science+Business Media, LLC, part of Springer Nature 2020

\section{Correction to: Current Cardiovascular Risk Reports https://doi.org/10.1007/s12170-020-00646-4}

We request that the following corrections be made in our article:

1. Author information located at the bottom title page should include:

Terrie-Ann S. Benjamin.

tsbenjamin@ healtheast.org
2. Extended information for author number 5, located under Affiliations, on the last page of the article - "Fairview Health Services and Medical Center, Houston, TX 77030, USA" should be replaced with:

M Health Fairview Heart Care, Saint Paul, MN 55102, USA.

Publisher's Note Springer Nature remains neutral with regard to jurisdictional claims in published maps and institutional affiliations.
The online version of the original article can be found at https://doi.org/ 10.1007/s12170-020-00646-4

Jacob Abraham

Jacob.Abraham@providence.org

1 Center for Cardiovascular Analytics, Research and Data Science (CARDS), Providence Heart Institute, Providence St. Joseph Health, 9427 SW Barnes Road, Suite 594, Portland, OR 97225, USA

2 Palmetto Health University of South Carolina Medical Group, Columbia, SC, USA

3 Krannert Institute of Cardiology, Indiana University School of Medicine, Indianapolis, IN 46202, USA

4 Houston Methodist Debakey Heart and Vascular Institute and Methodist J.C. Walter Jr. Transplant Center, Houston, TX 77030, USA
5 M Health Fairview Heart Care, Saint Paul, MN 55102, USA

6 Advanced Heart Failure and Transplantation Center, Harrington Heart and Vascular Institute, University Hospitals Cleveland Medical Center, Cleveland, OH 44106, USA

7 Sanford Cardiovascular Institute, Sanford University of South Dakota School of Medicine, Vermillion, SD 57069, USA

8 Sands Constellation Heart Institute, University of Rochester Medical Center, Rochester, NY 14642, USA

9 Austin Heart Hospital, Austin, TX 78756, USA

10 Minneapolis Heart Institute, Abbott Northwestern Hospital, Minneapolis, MN 55407, USA

11 Division of Cardiology, Scripps Clinic, San Diego, CA, USA 